\title{
Printed circuit board based segmented quadrupole ion guide
}

\author{
Maria Allers*, Florian Schlottmann, Manuel Eckermann, Stefan Zimmermann \\ Leibniz Universität Hannover, Institute of Electrical Engineering and Measurement Technology, \\ Department of Sensors and Measurement Technology, Appelstr. 9A, 30167 Hannover, Germany \\ *corresponding author: allers@geml.uni-hannover.de
}

\begin{abstract}
Collisional damping quadrupole ion guides have become popular tools in mass spectrometry. In such devices, ions are cooled by collisions with a buffer gas and focused to trajectories near the axis of the ion guide under the influence of a radio frequency (RF) field. This produces a narrow beam of lowenergy ions which can be transported with high efficiency. Typically, quadrupole ion guides are constructed of four parallel rods to which RF voltages are applied. To overcome the dampening of the axial velocity component resulting from collisions with neutral gas particles, an additional static axial field is provided by dividing the rods in several segments and applying an electric potential to each segment. However, this method is mechanically complex, requiring a precise alignment of all segments, and several separate connections for the DC und RF voltages to all segments. In this work, we present a simple and low-cost segmented quadrupole ion guide design that is based on standard printed circuit boards (PCB) including both the segmented electrodes as well as the signal distribution network. Furthermore, we present simulations of the ion movement inside this PCB quadrupole and experimentally evaluate the ion transfer. Our measurements show that the segmented PCB quadrupole with planar electrodes reaches similar ion transmissions in comparison with conventional quadrupole ion guides built from segmented circular rods.
\end{abstract}

\section{Introduction}

The linear quadrupole is widely used in many areas as a mass filter, ion guide or linear ion trap. Ideally, it consists of four parallel rods with hyperbolic cross-sections arranged in the corners of a square (see Figure 1a), to which DC and two RF voltages with opposite polarity are applied. The quadrupole field results from opposite electrodes being at the same phase, and adjacent electrodes being at opposite phase. At a given electrode distance, for particular magnitudes of the DC voltages as well as particular frequencies and amplitudes of the RF voltages, only ions of a specific mass-to-charge ratio can pass through the quadrupole to eventually reach an ion detector. Ions with lower or higher mass-to-charge collide with the rods to be discharged. By altering the voltages systematically, the transmitted massto-charge ratio can be scanned sequentially to generate a mass spectrum.

If a quadrupole is operated without DC voltage in RF-only mode, ions of a broad range of $\mathrm{m} / \mathrm{z}$ values will have stable trajectories in the quadrupole. Thus the quadrupole can be used as an ion guide for the transport of ions produced at atmospheric pressure into a mass analyzer operating under high vacuum. Quadrupole ion guides typically work in a pressure range from $1 \cdot 10^{-3} \mathrm{mbar}$ [1] to $0.1 \mathrm{mbar}$ [2] since ion-neutral gas collisions are required to effectively confine energetic ion motion in multipole RF devices, known as the collisional focusing effect [1]. This effect increases with gas pressure up to a point where ion scattering becomes the dominant process. The collisional focusing of ions in the quadrupole provides a narrow kinetic energy distribution of ions and a narrow spatial distribution of ions close to the quadrupole axis at the exit of the ion guide. Thus, a quadrupole ion guide allows to focus ions onto an exit aperture and acts as an excellent approximation to a point source of monoenergetic ions for a downstream mass analyzer. Hence, quadrupole ion guides have been successfully coupled to orthogonal injection time-of-flight (TOF) mass spectrometer, leading to 
improvements in sensitivity and resolution [2-4]. Furthermore, ion guides with collisional cooling have found widespread application in many other areas as collision or reaction cells [5-7].

The use of collisional focusing provides an ion beam of greatly improved quality regarding the kinetic energy distribution. However, an unwanted effect of collisional focusing in a quadrupole ion guide is that the ions undergo so many collisions that they are effectively brought to rest with no average forward motion before reaching the exit if the entrance velocity of the ions is insufficient to pass the quadrupole. Since in a conventional quadrupole there is no electric field in the axial direction, there is no force driving the ions towards the exit. Nonetheless, due to diffusion and space charge effects, ions eventually might reach the exit, but a significant time delay will occur, which causes problems in certain modes of operation [8].

In order to overcome these problems, several methods of generating an axial field directed along the axis of the quadrupole moving the ions actively towards the exit have been developed. A commonly used method is to divide the rods in several segments and apply a monotonically decreasing voltage to each segment $[9,10]$. This establishes an approximately uniform weak axial DC field, which causes the ions to drift through the quadrupole without influencing the focusing efficiency. However, this method is mechanically complex, requiring a precise alignment of all electrode segments, isolators and further mechanical parts as well as separate connections for the DC und RF voltages to all electrode segments, as indicated in Figure 1b. Another method of generating an axial field is the Linac ${ }^{\circledR}$ electrode geometry $[11,12]$. Here, one pair of cylindrical rods is tilted toward the entrance, the other pair toward the exit. This configuration results in one pair of opposing rods being closer to the axis at the entrance end, and the other pair of rods being closer to the axis at the exit end. With slightly different DC voltages applied to each rod pair, an axial potential gradient is established [13].

The aim of this work is to develop a simple and robust design for a quadrupole ion guide with axial electric field being appropriate to transport low $\mathrm{m} / \mathrm{z}$ ions at pressures in the range of $2 \cdot 10^{-2} \mathrm{mbar}$. While a quadrupole mass spectrometer requires accurately shaped and positioned electrodes to reach high resolutions, the confinement characteristics of quadrupole ion guides are particularly insensitive to the accuracy and precision of the surrounding electrodes [13]. An ideal quadrupole consists of four parallel electrodes with hyperbolic cross-sections. With new technologies as CNC manufacturing or other rapid prototyping technologies [14], such electrodes can be easily manufactured at reduced costs. However, most commonly quadrupole electrodes are in the form of circular rods. Circular electrodes generate a field that consists of a hyperbolic component (second order) with a number of higher order components superimposed. However, with the correct ratio of electrode radius $r$ to central field radius $r_{0}$, a close approximation to the ideal hyperbolic field is achievable [15-18]. Even further, any electrode geometry that generates a saddle point of the electric potential is capable to dynamically confine charged particles. The lowest order coefficient of a multipole expansion around the saddle point will be quadrupolar. Providing that all higher order terms remain sufficiently small, the particle's trajectory in the vicinity of this point can be approximated by that in a harmonic pseudopotential [19]. This allows particle confinement even with unusual electrode geometries like planar electrodes [20-22] or rectangular electrodes [23], and enables the realization of quadrupole ion guides, filters and traps based on different design concepts and construction technologies [14,24,25]. In particular, planar electrodes are easy to realize using printed circuit boards (PCBs). This technology allows the design and rapid fabrication of different electrode geometries at low cost. Thus, in recent years, various ion optical devices based on PCBs have been presented. For example, Smith and coworkers introduced structures for lossless ion manipulation (SLIM) consisting of electrodes patterned on PCBs [26-28]. In these devices, RF ion confinement in conjunction with DC potentials is used to manipulate ions in multiple dimensions at pressures in the low Torr range. Other groups [2931] described PCB based planar ion traps for mass analysis at high vacuum conditions. 
However, a PCB based quadrupole ion guide being appropriate to transport low $\mathrm{m} / \mathrm{z}$ ions at pressures in the range of $2 \cdot 10^{-2} \mathrm{mbar}$ has not been presented yet. As stated above, in this pressure range, an additional axial electric field is needed to transport ions to the exit of the quadrupole. Using PCB technology, an axial electric field is easy to implement by segmenting the electrodes in axial direction and applying a monotonically decreasing potential to each segment. In this work, such a segmented quadrupole ion guide is presented. It is entirely manufactured from standard PCBs. Figure $1 \mathrm{c}$ shows the quadrupole design concept. It consists of only seven parts: four identical electrode PCBs forming the sidewalls of the ion guide, and three PCB adapters for mounting. The use of PCB technology is beneficial, as the electrode PCBs not only include 64 electrode segments on the inside, but also the signal distribution network, the electrical components and the connections to the DC and RF power supplies on the outside of the quadrupole. Thus, this design is mechanically simple, easy to fabricate and easy to assemble.

In this work, the analytical performance of the presented PCB quadrupole ion guide will be evaluated by simulations and measurements.

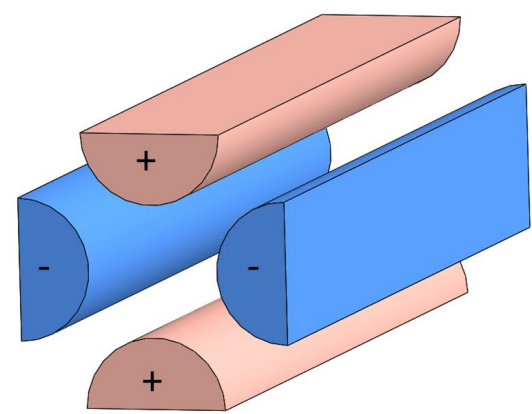

a) RF-only quadrupole ion guide

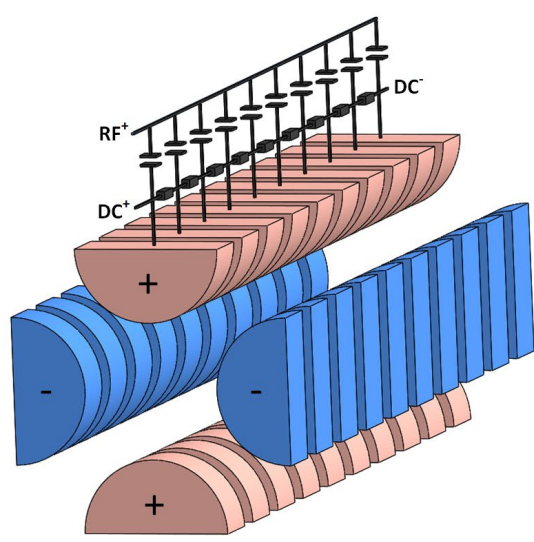

b) Segmented quadrupole ion guide

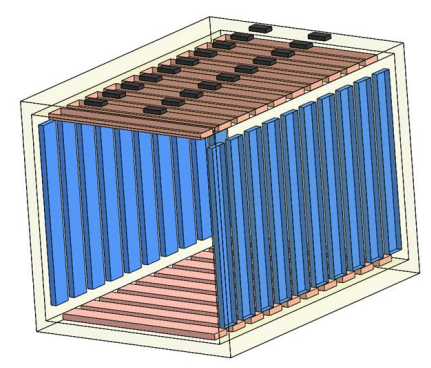

c) Segmented PCB quadrupole ion guide

Figure 1: Different types of quadrupole ion guides. a) Quadrupole ion guide in RF-only mode: four parallel rods with RF voltages applied to the electrodes, b) segmented rods with separate connections for the axial $D C$ und $R F$ voltages to all segments, as shown for the upper rod, c) PCBs with thin planar segmented electrodes on the inside and electrical components on the outside of the quadrupole.

\section{Theoretical considerations}

To theoretically evaluate the analytical performance of the presented PCB quadrupole ion guide with planar electrodes in contrast to a quadrupole with hyperbolic electrodes, ion trajectories inside a quadrupolar field are considered in detail.

Assuming an ideal hyperbolic electrode geometry and neglecting collisions of ions with neutral background molecules, the ion trajectories can be described analytically.

For mass analysis, the electrical voltage applied to the quadrupole rods is given by

$$
\Phi_{0}(t)=U-V_{R F} \cos \omega t
$$

$\Phi_{0}(t)$ consists of a DC portion $U$ and an RF portion with amplitude $V_{R F}$ and angular frequency $\omega$. In this case, the quadrupole potential is

$$
\Phi(x, y, t)=\left(\frac{x^{2}-y^{2}}{r_{0}^{2}}\right)\left(U-V_{R F} \cos \omega t\right)
$$


where $x$ and $y$ are Cartesian coordinates in the axial cross-section [32]. The point $(x, y)=(0,0)$ is the center of the quadrupole. The parameter $r_{0}$ is the distance from the center to an electrode. This quadrupole field deflects the ions in the $x$ and $y$ directions, forcing them onto helical trajectories around the $z$ axis through the mass filter. The equations of motion in $x$-direction for an ion with mass $m$ and charge $z \cdot e$ can be described by the differential equation (3) [32].

$$
m \frac{d^{2} x}{d t^{2}}=-\frac{2 z e x}{r_{0}{ }^{2}}\left(U-V_{R F} \cos \omega t\right)
$$

Introducing the variables

$$
a_{x}=\frac{8 z e U}{m r_{0}{ }^{2} \omega^{2}}, \quad q_{x}=\frac{4 z e V_{R F}}{m r_{0}^{2} \omega^{2}}, \quad \xi=\frac{\omega t}{2}
$$

Equation (3) becomes

$$
\frac{d^{2} x}{d \xi^{2}}+\left(a_{x}-2 q_{x} \cos 2 \xi\right) x=0
$$

Equation (5) is the Mathieu equation [32]. The parameters $a_{x}$ and $q_{x}$ are characteristic for the given application. For a quadrupole, $a_{x}$ describes the influence of the DC field and $q_{x}$ describes the interaction of the ions with the RF field. The solution of the Mathieu equation is characterized by regions of stability and instability according to whether the amplitude of the ion motion grows exponentially or remains confined in space. For quadrupole ion guides, the DC voltage $U$ and the parameter $a_{x}$ are zero. Thus, stability criteria can be expressed in terms of the non-dimensional parameter $q_{x}$. All trajectories of the ions where $q_{x}<0.908$ will be stable (other, smaller regions of stability in $q$-space are ignored here). According to Equation (4), these will be all masses which satisfy Equation (6).

$$
m>\frac{4 z e V_{R F}}{0.908 \cdot r_{0}^{2} \cdot \omega^{2}}
$$

The quadrupole thus acts as a high pass filter in this operating mode. Increasing the RF amplitude $V_{R F}$, the low-mass cut-off also increases. Furthermore, it has to be taken into account that not all combinations of initial position and initial radial velocity are transmitted even though the condition $q_{x}<0.908$ is satisfied. For a given RF phase, combinations of initial position $(x, y)$ and radial velocity $v_{x}=\frac{d x}{d t}, v_{y}=\frac{d y}{d t}$ that are transmitted, fall within ellipses in the $x-v_{x}$ and the $y-v_{y}$ planes [32].The area of an ellipse is the "acceptance" for that particular phase. These ellipses rotate with changing phase. The region where the ellipses overlap for all phases gives the acceptance in $x$ or $y$ for $100 \%$ transmission regardless of RF phase [32]. Thus, ions have to enter the quadrupole in this region close to the center of the quadrupole and ideally move parallel to the axis of the rod. The larger the inner radius of the quadrupole $r_{0}$ and the longer the quadrupole, the easier it is to fulfill these stability conditions.

However, the above stated analytical description of the ion trajectories in a quadrupole is only valid for an ideal hyperbolic electrode geometry and for neglecting collisions of ions with neutral background molecules. To estimate the transmission efficiency of the presented PCB quadrupole ion guide with planar electrodes at a pressure of $2 \cdot 10^{-2} \mathrm{mbar}$ in comparison to an ideal quadrupole with hyperbolic electrodes at a pressure of $2 \cdot 10^{-2}$ mbar, numerical simulations are necessary.

The transmission efficiency is determined by the focusing ability of the quadrupole. To ensure a high transmissions of ions through a downstream exit aperture, ions need to be focussed in the centre of the quadrupole. Thus, in this work, the transmission efficiency is estimated by considering the 
simulated focusing efficiency for ions in a simple $2 \mathrm{D}$ cross section of an infinitely long quadrupole. The ion trajectories have been simulated in COMSOL Multiphysics 5.4 using the Electric Currents and the Particle Tracing module. Collisions of the ions with the background gas are included enabling elastic collisions in the Particle Tracing module and assuming a collision cross section of $5 \cdot 10^{-19} \mathrm{~m}^{2}$ for the collision between protonated water clusters $\mathrm{H}^{+}\left(\mathrm{H}_{2} \mathrm{O}\right)_{n}$, considered as model ions, and nitrogen, according to [33]. The influence of gas flow and Coulomb repulsion is neglected. Due to given geometric constraints in the experimental setup, the inner diameter of the geometry is set to $6 \mathrm{~mm}$. A RF voltage with variable amplitude $V_{R F}$ at a frequency $f$ of $5 \mathrm{MHz}$ is applied to the electrodes. The time step taken by the solver is $4 \mathrm{~ns}$.

In Figure 2, the initial ion distribution at $\mathrm{t}=0 \mathrm{~s}$ is shown. 1000 ions are uniformly distributed in a cross section between the electrodes in a circle with $5 \mathrm{~mm}$ diameter. In the simulation, the ion distribution is considered over time. The amount of ions inside a circle with $1 \mathrm{~mm}$ diameter after $100 \mu \mathrm{s}$ simulated RF confinement serves as a measure for the focusing efficiency and consequently the transmission efficiency of the quadrupole. Only comparing the focusing ability of the geometries, the initial axial and radial ion velocity components are set to zero.
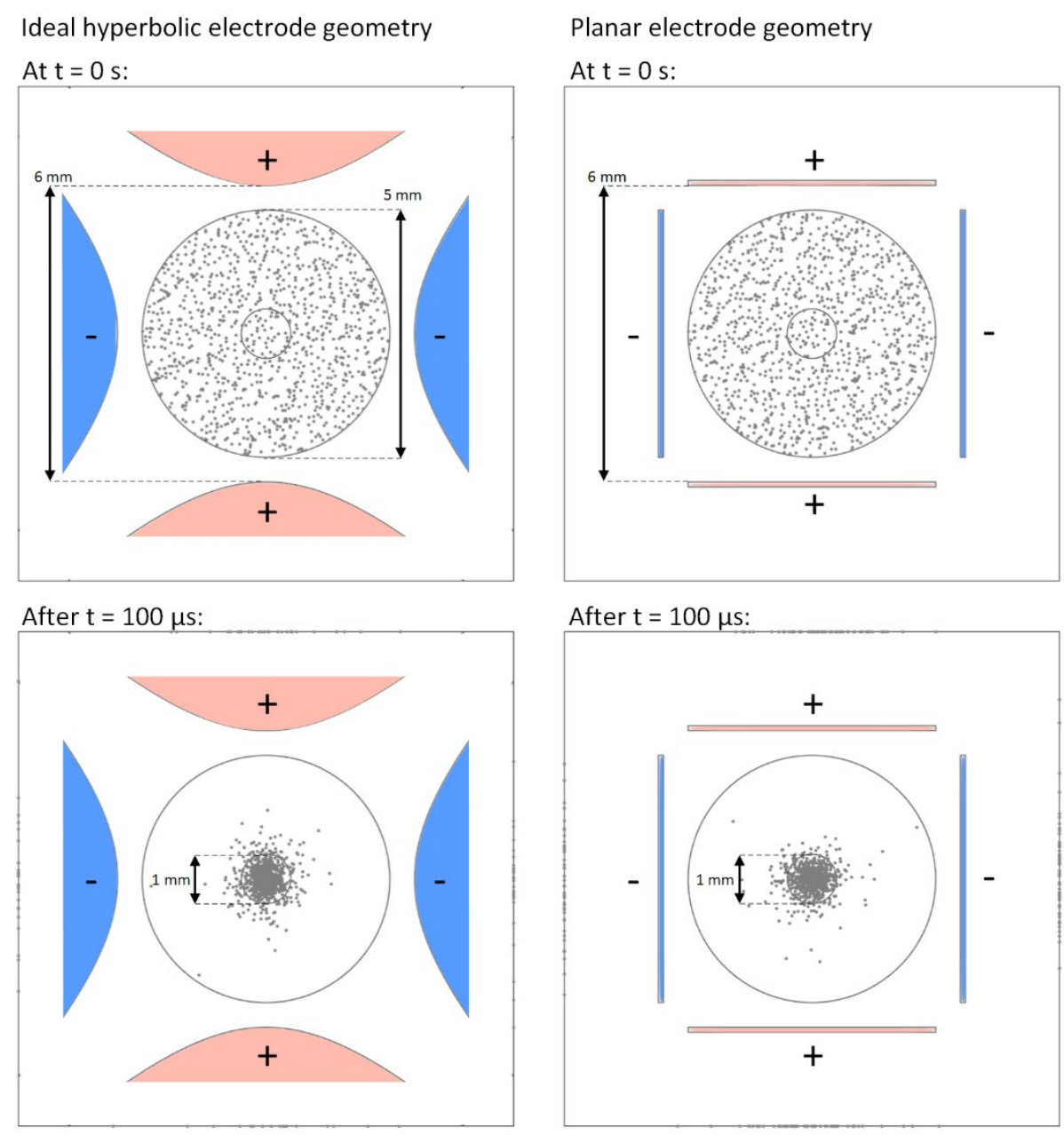

Figure 2: Simulated 2D ion distribution between the quadrupole electrodes at $t=0 \mathrm{~s}$ and after $t=100 \mu \mathrm{s} R \mathrm{~F}$ confinement for the ideal hyperbolic electrode geometry (left) and the planar electrode geometry (right). lon mass: $30 \mathrm{~m} / \mathrm{z}$, RF voltage amplitude: $90 \mathrm{~V}$ zero-to-peak, RF frequency: $5 \mathrm{MHz}$, pressure: $2 \cdot 10^{-2} \mathrm{mbar}$.

Figure 3a shows the simulated focusing efficiencies for masses between $10 \mathrm{~m} / \mathrm{z}$ and $150 \mathrm{~m} / \mathrm{z}$ dependent on the RF voltage amplitude using an ideal hyperbolic electrode geometry. Particularly low mass ions suffer from discrimination when increasing the RF voltage amplitude. For example, for RF 
voltage amplitudes exceeding $100 \mathrm{~V}$ zero-to-peak, the trajectories of ions with a mass of $10 \mathrm{~m} / \mathrm{z}$ become unstable. Furthermore, at RF voltage amplitudes exceeding $250 \mathrm{~V}$ zero-to-peak, ions with a mass of $20 \mathrm{~m} / \mathrm{z}$ are no longer focused.

In Figure $3 b$, the simulated results for the planar electrode geometry are shown. As stated above, to reach high mass resolution, a quadrupole mass filter requires a preferably ideal quadrupolar field, which is not achievable with planar electrodes. In contrast, the confinement characteristics of a quadrupole ion guide is particularly insensitive to the ideality of the electric field. This can be confirmed by the results in Figure $3 \mathrm{~b}$. Both geometries show comparable focussing behaviour. The ideal hyperbolic geometry only reaches a slightly better focusing efficiency compared to the planar geometry. The focusing efficiency is only around 10 to $20 \%$ higher. Thus, in our application as a pure ion guide, the performance of the PCB quadrupole with planar electrodes is sufficient.
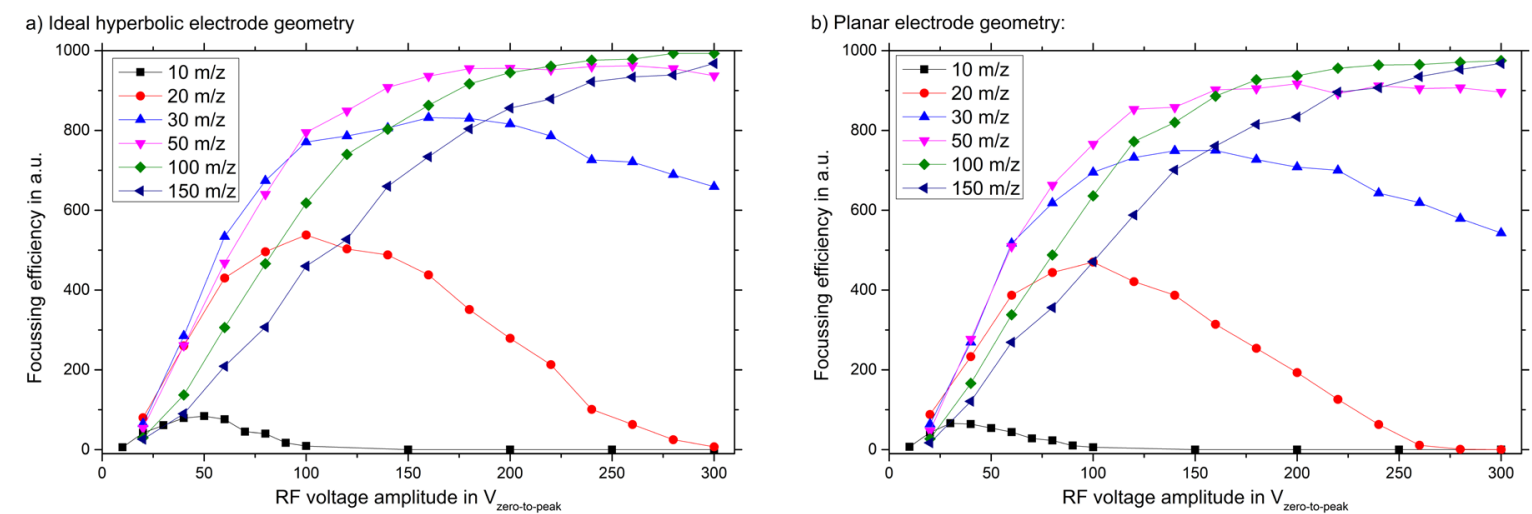

Figure 3: Simulated focusing efficiencies for masses between $10 \mathrm{~m} / \mathrm{z}$ and $150 \mathrm{~m} / \mathrm{z}$ dependent on the RF voltage amplitude at a frequency of $5 \mathrm{MHz}$ and a pressure of $2 \cdot 10^{-2}$ mbar. a) Ideal hyperbolic electrode geometry, b) planar electrode geometry.

\section{Segmented quadrupole ion guide based on PCB}

As mentioned above, the segmented quadrupole ion guide is entirely manufactured from standard PCBs. The PCBs used in this work are made of a glass-reinforced epoxy laminate (FR4), which is laminated with a $35 \mu \mathrm{m}$ thin film of copper. The copper can be structured using lithographic processes to generate the necessary geometries for the electrodes with high precision. The length of the quadrupole is specified by the dimensions of the surrounding vacuum chamber. Each of the four side elements of the quadrupole ion guide is equipped with $642 \mathrm{~mm}$ wide electrodes. These electrodes have a spacing of $0.2 \mathrm{~mm}$, resulting in a total length of the quadrupole ion guide of $141.7 \mathrm{~mm}$. Due to the given geometry restrictions, the inner diameter of the ion guide is $6 \mathrm{~mm}$. Between the electrodes, holes of $0.8 \mathrm{~mm}$ width allow for evacuation of the ion guide to $2 \cdot 10^{-2}$ mbar. Before assembly all PCBs are cleaned and evacuated at $80^{\circ} \mathrm{C}$ to prevent any outgassing during operation. The electrode design is shown in detail in Figure $4 a$.

The concept of the PCB quadrupole ion guide is similar to the PCB ion mobility spectrometer described in [34] and the PCB ion funnel described in [35]. Basically, the PCB quadrupole ion guide consists of four identical $\mathrm{PCB}$ including the electrodes and the signal distribution network as well as three additional PCBs for mounting the quadrupole in the vacuum chamber. The electrode PCBs are adhered with HUNTSMAN Araldite 2014-1 forming the sidewalls of the ion guide, as depicted in Figure 4b. The adhesive Araldite is well suited for vacuum applications. In Figure 4c, the completely assembled PCB quadrupole is shown. 


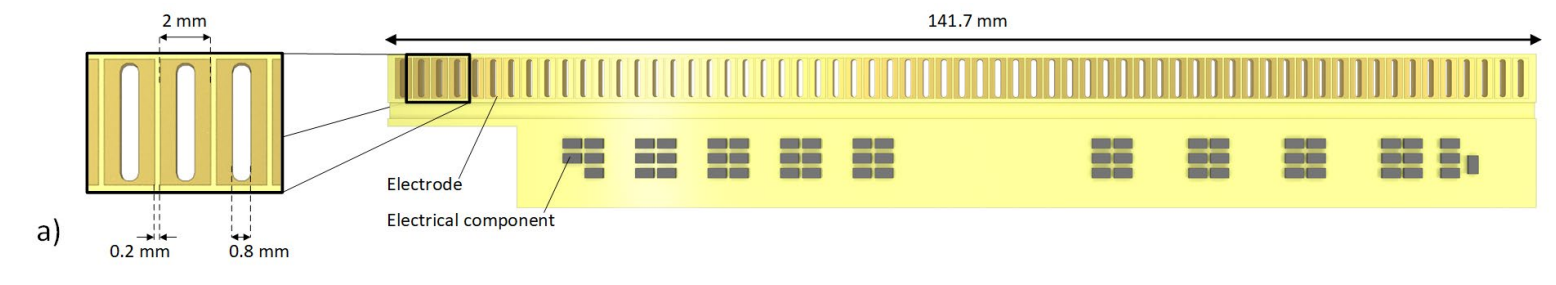

b)
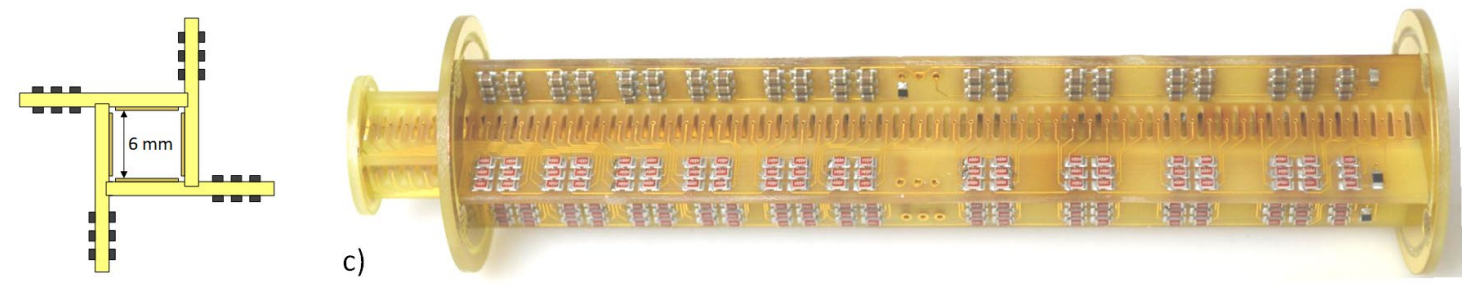

Figure 4: a) One quadrupole side element including 64 electrodes, holes for evacuation and the signal distribution network, b) schematic of the assembled quadrupole side elements, c) photo of the PCB quadrupole ion guide including PCBs for mounting.

On the backside of the PCBs, the electrodes are connected to the signal distribution network and via connectors to the RF and DC power supplies. The series resistor chain consists of $4.99 \mathrm{MOhm}$ resistors from BOURNS with a working voltage up to $400 \mathrm{~V}$. This DC voltage divider provides a linear DC voltage gradient to the electrodes along the quadrupole axis. Two RF voltages that are opposite in phase are connected to adjacent electrodes via $2700 \mathrm{pF}$ coupling capacitors from KEMET with a voltage rating of $1 \mathrm{kV}$. The sine waveform for generating the RF voltages $V_{R F}{ }^{+}$and $V_{R F}{ }^{-}$is provided by a signal generator (RIGOL DG4062). The output of the signal generator is amplified by a $7 \mathrm{~W}$ RF power amplifier (ENI 607L01). The necessary $180^{\circ}$ phase shift of the RF voltages is generated by using a 1:4 BalUn, which transforms the unbalanced signal oscillating relative to ground in two balanced signals with identical amplitudes but opposite in phase. The BalUn is connected to two custom built Hartley oscillators forming resonant circuits in combination with the coupling capacitors of the quadrupole. The resonance frequency is about $5 \mathrm{MHz}$.

\section{Experimental setup}

In order to experimentally evaluate the ion transmission of our PCB quadrupole ion guide, the ion guide is integrated in an intermediate pressure stage at $2 \cdot 10^{-2} \mathrm{mbar}$ to transport ions generated at $15 \mathrm{mbar}$ into a high vacuum region at $6 \cdot 10^{-6} \mathrm{mbar}$, as shown in Figure 5 . Basically, the setup consists of five primary components: (1) a corona discharge ionization source; (2) a drift tube; (3) an ion funnel to focus ions onto the first aperture with $0.7 \mathrm{~mm}$ in diameter, (4) the segmented quadrupole ion guide to focus ions onto the second aperture with $1 \mathrm{~mm}$ in diameter, and (5) the high vacuum region with a Faraday detector right behind the second aperture to measure the transmitted ion current. The Faraday detector is connected to a current amplifier (FEMTO DLPCA-200, gain 10 $\mathrm{V} / \mathrm{A}, 10 \mathrm{~Hz}$ ).

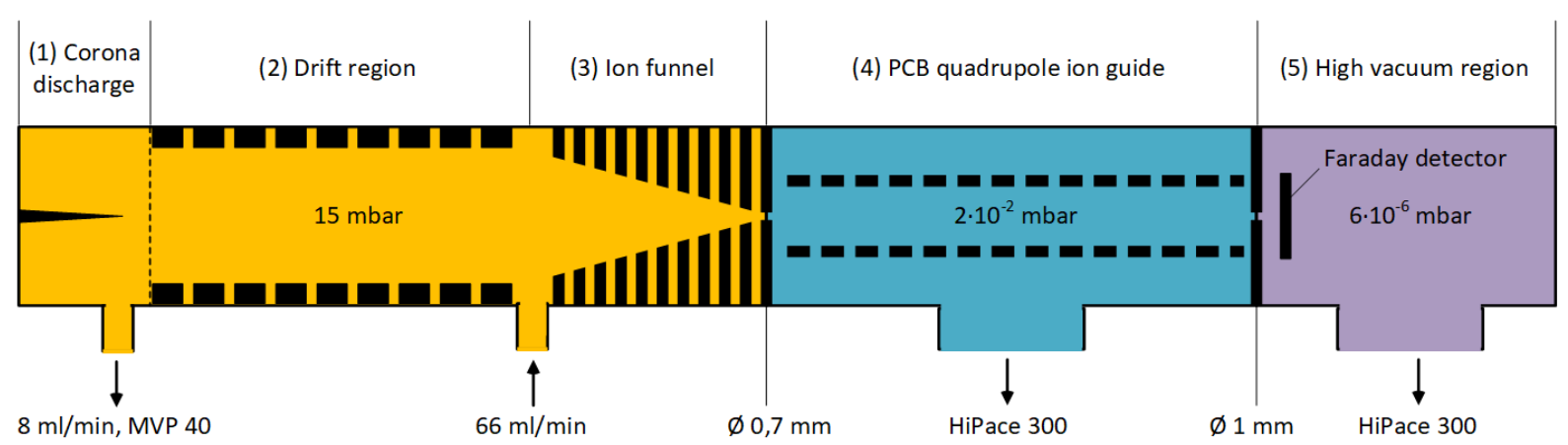

Figure 5: Experimental setup: (1) Corona discharge ionization, (2) drift tube, (3) ion funnel, (4) PCB quadrupole ion guide, (5) high vacuum region. 
Both the drift tube and the ion funnel are operated at 15 mbar and evacuated via a membrane pump (MVP 40, Pfeiffer Vacuum). A gas dosing valve (EVN 116, Pfeiffer Vacuum) is used to adjust the pumping rate. The pressure within the drift tube is monitored via a capacitive pressure gauge (Pfeiffer Vacuum, CMR 362). A constant gas flow of $66 \mathrm{mls}^{1} / \mathrm{min}$ of dry clean air is introduced into the drift tube by a mass flow controller (Bronkhorst). A corona discharge needle (corona needle APCl, Agilent) in point-to-plane geometry is used to generate the primary ions. The drift tube consists of metallic guard rings with a constant voltage applied, leading to a homogeneous electric field at a reduced electric field strength of $100 \mathrm{Td}^{2}$ within the drift tube. In future setups, this drift tube might be replaced by a HiKE-IMS [36-39] to realize a HiKE-IMS-MS for further investigation of the gas phase ion chemistry in HiKE-IMS. The drift tube is coupled to a PCB ion funnel [35] permanently operating at a reduced DC electric field strength of $30 \mathrm{Td}$ and a RF voltage amplitude of $190 \mathrm{~V}$ zero-to-peak at $4.16 \mathrm{MHz}$ for generating a constant ion current of $1 \mathrm{nA}$ [35]. The ion funnel focuses the ions onto the first aperture and transmits them into the intermediate pressure stage with $2 \cdot 10^{-2} \mathrm{mbar}$, which is formed by a DN 40, CF 6-way cross and evacuated via a turbopump (HiPace 300, Pfeiffer Vacuum). The pressure within the intermediate stage is monitored via an active pirani/cold cathode pressure gauge (PKR 251, Pfeiffer Vacuum). Our PCB quadrupole ion guide is placed inside the DN 40, CF 6-way cross to transport ions from the first aperture through the second aperture into the high vacuum region at $6 \cdot 10^{-6} \mathrm{mbar}$.

The electric circuit driving the segmented electrodes of the PCB quadrupole ion guide is shown in Figure 6. A DC voltage $U_{\text {quad }}$ provides a defined electric field along the axis of the quadrupole. For ion injection into the quadrupole, an additional DC voltage $U_{i n j}$ is applied between the first aperture and the first quadrupole segment. Furthermore, in order to shield the quadrupole by the surrounding ground potential of the vacuum chamber, the quadrupole ion guide is enclosed by a stainless steel grid connected to the potential of the first electrode segment.

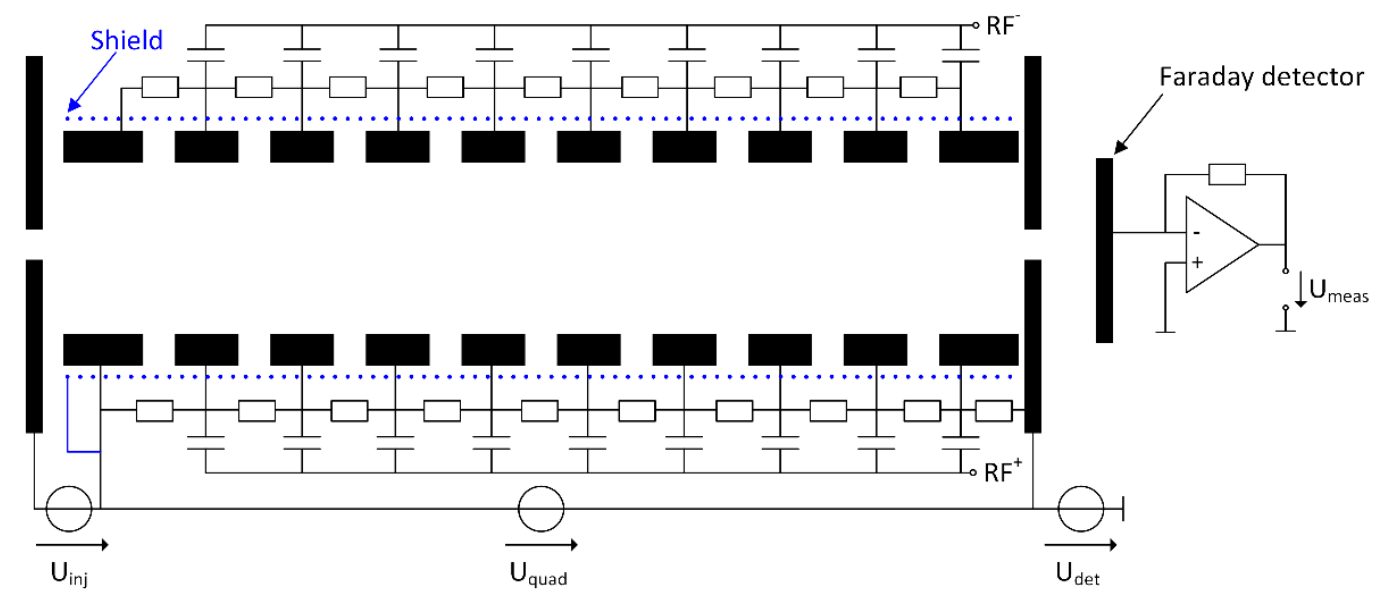

Figure 6: Electric overview

The drift tube, ion funnel and quadrupole DC voltages are provided by custom-built electronics. The output of the current amplifier is connected to a custom-built data acquisition system. The applied RF voltage amplitudes are monitored by an oscilloscope (Agilent InfiniiVision DSO-X-4104A). Table 1 summarizes the operating parameters.

\footnotetext{
${ }^{1}$ Gas volume given for 1013 mbar and $293.15 \mathrm{~K}$

${ }^{2}$ Townsend (Td): Unit of the reduced electric field strength (ratio between the electric field $E$ and the number density of the neutral $N) .1 \mathrm{Td}=10^{-21} \mathrm{~V} \cdot \mathrm{m}^{2}$
} 


\begin{tabular}{|l|l|}
\hline Parameter & Value \\
\hline Length of drift tube & $269 \mathrm{~mm}$ \\
\hline Length of ion funnel & $69 \mathrm{~mm}$ \\
\hline Length of quadrupole & $142 \mathrm{~mm}$ \\
\hline $\begin{array}{l}\text { RF voltage frequency of ion } \\
\text { funnel }\end{array}$ & $4.159 \mathrm{MHz}$ \\
\hline $\begin{array}{l}\text { Zero-to-peak RF voltage of } \\
\text { ion funnel }\end{array}$ & $190 \mathrm{~V}$ \\
\hline $\begin{array}{l}\text { RF voltage frequency of } \\
\text { quadrupole }\end{array}$ & $5.2 \mathrm{MHz}$ \\
\hline $\begin{array}{l}\text { Zero-to-peak RF voltage of } \\
\text { the quadrupole }\end{array}$ & $0-250 \mathrm{~V}$ \\
\hline Corona voltage & $1350 \mathrm{~V}$ \\
\hline $\begin{array}{l}\text { Reduced electric } \mathrm{DC} \text { field in } \\
\text { drift region }\end{array}$ & $100 \mathrm{Td}$ \\
\hline $\begin{array}{l}\text { Reduced electric } \mathrm{DC} \text { field in } \\
\text { ion funnel }\end{array}$ & $30 \mathrm{Td}$ \\
\hline $\begin{array}{l}\text { Quadrupole injection } \\
\text { voltage } \mathrm{U}_{\text {inj }}\end{array}$ & $0-30 \mathrm{~V}$ \\
\hline Quadrupole voltage $\mathrm{U}_{\text {quad }}$ & $0-9 \mathrm{~V}$ \\
\hline Detection voltage $\mathrm{U}_{\text {det }}$ & $100 \mathrm{~V}$ \\
\hline Dew point of drift gas & $-90^{\circ} \mathrm{C}$ \\
\hline Drift gas flow & $66 \mathrm{mls} / \mathrm{min}$ \\
\hline $\begin{array}{l}\text { Operating pressure in the } \\
\text { drift tube and ion funnel }\end{array}$ & $15 \mathrm{mbar}$ \\
\hline $\begin{array}{l}\text { Operating pressure in the } \\
\text { low-pressure region }\end{array}$ & $2 \cdot 10^{-2} \mathrm{mbar}$ \\
\hline $\begin{array}{l}\text { Operating pressure in the } \\
\text { high vacuum region }\end{array}$ & $6 \cdot 10^{-6} \mathrm{mbar}$ \\
\hline Operating temperature & $30^{\circ} \mathrm{C}$ \\
\hline
\end{tabular}

\section{Results und discussion}

Under the conditions stated above, it can be assumed that the positive ion population in drift region and ion funnel generated by the corona discharge ionization is dominated by $n=1$ and $n=2$ water clusters $\left(\mathrm{H}_{2} \mathrm{O}\right)_{n} \mathrm{H}^{+}$, nitrogen oxide $\mathrm{NO}^{+}$and oxygen $\mathrm{O}_{2}{ }^{+}[40-42]$. Thus, ions with small masses between $19 \mathrm{~m} / \mathrm{z}$ and $55 \mathrm{~m} / \mathrm{z}$ enter the quadrupole. In the following, the transmission efficiency of our segmented PCB quadrupole ion guide will be evaluated for these ions. Therefore, the effect of the RF voltage amplitude $V_{R F}$, the injection voltage $U_{i n j}$ and the quadrupole voltage $U_{\text {quad }}$ on the transmitted ion current is experimentally investigated. In the current experimental setup, the RF voltage amplitude $V_{R F}$ is restricted to $250 \mathrm{~V}$ zero-to-peak due to the limited output of the RF power amplifier used in this work. For the transmission of the low mass ions considered in this work, a RF amplitude of $250 \mathrm{~V}$ zeroto-peak is sufficient. However, in other applications, elevated RF amplitudes might be required to transmit ions with higher masses, e.g. $1000 \mathrm{~V}$ zero-to-peak in ESI applications $[3,43]$. Nonetheless, by adapting the RF power supply, the mounted capacitors and the resistors, the RF voltage amplitude could be raised to enable the application of the PCB quadrupole ion guide in these fields.

Figure 7 shows the effect of the RF voltage amplitude $V_{R F}$ on the ion current. As predicted by the theoretical considerations, the ion current increases with increasing RF voltage amplitude $V_{R F}$. However, in contrast to the simulated results, a minimum RF voltage amplitude $V_{R F}$ of $125 \mathrm{~V}$ zero-topeak is required to effectively focus and transport ions to the ion guide exit aperture. This deviation is 
due to the exclusion of a) field inhomogeneities at the entrance and the exit of the quadrupole and $b$ ) the initial radial and axial ion velocities caused by the gas expansion in the simulations.

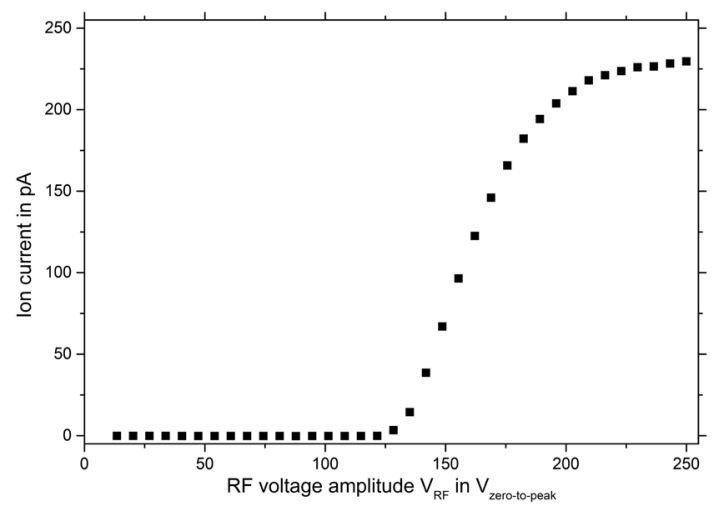

Figure 7: Measured ion current in dependent on the $R F$ voltage amplitude $V_{R F} . U_{i n j}=20 \mathrm{~V}, U_{\text {quad }}=7 \mathrm{~V}$.

As mentioned above, at a pressure of $2 \cdot 10^{-2} \mathrm{mbar}$, ions require kinetic energies in axial direction to reach the exit of the quadrupole ion guide. This effect can be observed when varying the injection voltage $U_{i n j}$ or the quadrupole voltage $U_{\text {quad. }}$. Both voltages affect the axial kinetic energy of the ions. In Figure 8a, the influence of the injection voltage $U_{\text {inj }}$ on the ion transmission for $U_{\text {quad }}=0 \mathrm{~V}$ is shown. A minimum injection voltage $U_{i n j}$ of $15 \mathrm{~V}$ is required to measure an ion current at the Faraday detector. A saturation is reached for injection voltages $U_{i n j}$ exceeding $30 \mathrm{~V}$. Hence, at high injection voltages $U_{i n j,}$ some ions gain enough initial kinetic energy to reach the exit of the quadrupole without the need of an additional axial field provided by the quadrupole voltage $U_{\text {quad. }}$. However, solely applying an injection voltage $U_{i n j}$, the maximum measured ion current is just $5 \mathrm{pA}$. Significantly higher ion transmission efficiencies can be reached by applying an additional quadrupole voltage $U_{\text {quad }}$ of just a few volts, leading to an axial field that moves the ions to the exit of the quadrupole. As shown in Figure $8 \mathrm{~b}$, the additional quadrupole voltage $U_{\text {quad }}$ results in ion currents exceeding $250 \mathrm{pA}$.

a)

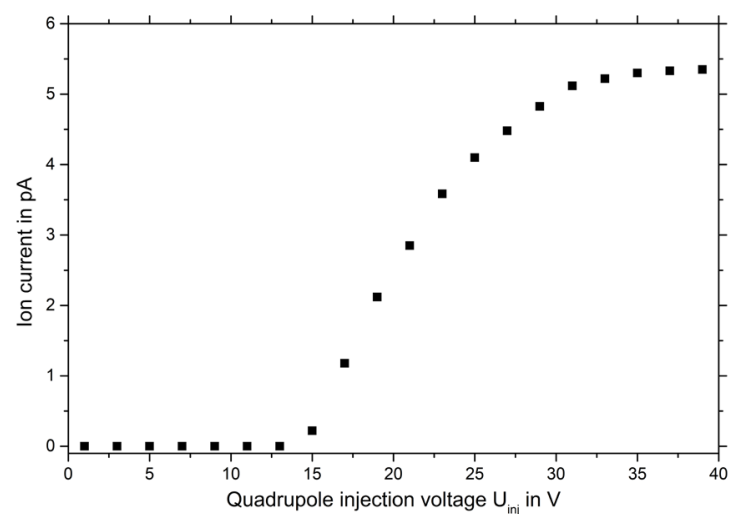

b)

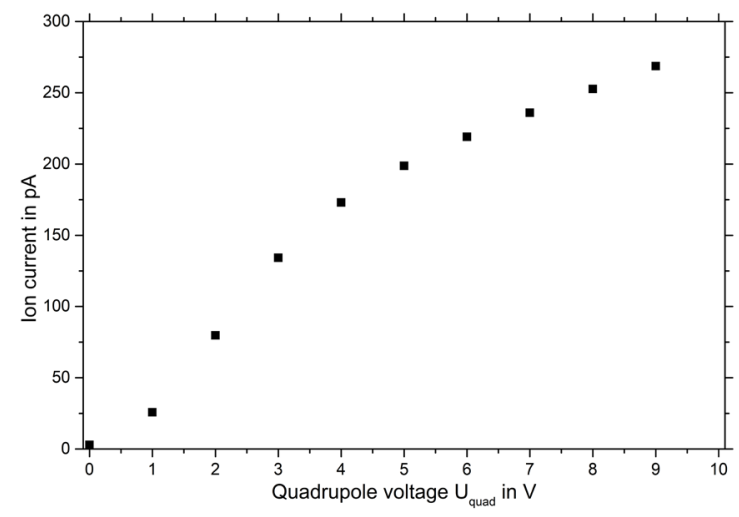

Figure 8: a) Measured ion current dependent on the quadrupole injection voltage $U_{\text {inj. }} U_{\text {quad }}=0 \mathrm{~V}, V_{R F}=250 \mathrm{~V}$ zero-to-peak, b) measured ion current dependent on the quadrupole voltage $U_{\text {quad. }} U_{\text {inj }}=21 \mathrm{~V}, V_{R F}=250 \mathrm{~V}$ zero-to-peak.

These measurements show that our segmented PCB quadrupole ion guide is appropriate to focus and transmit ions at a pressure of $2 \cdot 10^{-2}$ mbar. By varying the quadrupole voltage $U_{\text {quad, }}$, the axial kinetic energy of the ions can be controlled. The additional axial electric field helps to transport more ions to the exit of the quadrupole. Under the conditions stated above, the ion current being transmitted by the ion funnel through the first aperture and entering the quadrupole is $1060 \mathrm{pA}$ [35]. In this work, a maximum ion current through the second aperture of $270 \mathrm{pA}$ is measured. Thus, the PCB quadrupole ion guide reaches a transmission efficiency of about $25 \%$ for small masses between $19 \mathrm{~m} / \mathrm{z}$ and $55 \mathrm{~m} / \mathrm{z}$. Douglas and French [1] measured a transmission efficiency of $18 \%$ through a $1 \mathrm{~mm}$ aperture for ions 
of $\mathrm{m} / \mathrm{z} 23$ - 100 using a conventional quadrupole in RF-only mode at a pressure of $0.8 \cdot 10^{-2} \mathrm{mbar}$. Thus, our PCB quadrupole ion guide performs similarly and represents an easy to manufacture low-cost alternative to conventional segmented quadrupole ion guides.

\section{Conclusion}

In this work, we present a segmented quadrupole design entirely based on standard PCB technology. The quadrupole is mechanically simple, easy to fabricate and easy to assemble at low cost. It consists of only seven parts: four electrode PCBs including the signal distribution circuit with electrical components forming the sidewalls of the quadrupole, and three PCB adapters for mounting. The PCB quadrupole operates at a pressure of $2 \cdot 10^{-2} \mathrm{mbar}$ and effectively focuses and transports ions. The measurement results show that such a segmented $\mathrm{PCB}$ quadrupole with planar electrodes reaches similar ion transmission in comparison with conventional quadrupole ion guides built from segmented circular rods.

\section{Acknowledgement}

This work was funded by the Deutsche Forschungsgemeinschaft (DFG, German Research Foundation) - ZI 1288/8-1 


\section{References}

[1] D. J. Douglas, J. B. French (1992): Collisional focusing effects in radio frequency quadrupoles, J Am Soc Mass Spectrom 3, 398-408; doi: 10.1016/1044-0305(92)87067-9.

[2] A. N. Krutchinsky, I. V. Chernushevich, V. L. Spicer, W. Ens, K. G. Standing (1998): Collisional damping interface for an electrospray ionization time-of-flight mass spectrometer, J Am Soc Mass Spectrom 9, 569-579; doi: 10.1016/S1044-0305(98)00027-0.

[3] A. N. Krutchinsky, A. V. Loboda, V. L. Spicer, R. Dworschak, W. Ens, K. G. Standing (1998): Orthogonal injection of matrix-assisted laser desorption/ionization ions into a time-of-flight spectrometer through a collisional damping interface, Rapid Commun. Mass Spectrom. 12, 508518; doi: 10.1002/(SICI)1097-0231(19980515)12:9<508:AID-RCM197>3.0.CO;2-L.

[4] P. Sulzer, E. Hartungen, G. Hanel, S. Feil, K. Winkler, P. Mutschlechner, S. Haidacher, R. Schottkowsky, D. Gunsch, H. Seehauser, M. Striednig, S. Jürschik, K. Breiev, M. Lanza, J. Herbig, L. Märk, T. D. Märk, A. Jordan (2014): A Proton Transfer Reaction-Quadrupole interface Time-OfFlight Mass Spectrometer (PTR-QiTOF), Int. J. Mass Spectrom. 368, 1-5; doi: 10.1016/j.ijms.2014.05.004.

[5] Z. Du, D. J. Douglas (1999): A novel tandem quadrupole mass analyzer, J Am Soc Mass Spectrom 10, 1053-1066; doi: 10.1016/S1044-0305(99)00094-X.

[6] D. J. Douglas (1998): Applications of collision dynamics in quadrupole mass spectrometry, J Am Soc Mass Spectrom 9, 101-113; doi: 10.1016/S1044-0305(97)00246-8.

[7] S. D. Tanner, V. I. Baranov, D. R. Bandura (2002): Reaction cells and collision cells for ICP-MS: a tutorial review, Spectrochimica Acta Part B: Atomic Spectroscopy 57, 1361-1452; doi: 10.1016/S0584-8547(02)00069-1.

[8] M. Morris, P. Thibault, R. K. Boyd (1994): Characterization of a high-pressure quadrupole collision cell for low-energy collision-indneed dissociation, J Am Soc Mass Spectrom 5, 10421063; doi: 10.1016/1044-0305(94)85066-6.

[9] G. Javahery, B. Thomson (1997): A segmented radiofrequency-only quadrupole collision cell for measurements of ion collision cross section on a triple quadrupole mass spectrometer, J Am Soc Mass Spectrom 8, 697-702; doi: 10.1016/S1044-0305(97)00027-5.

[10] A. Dodonov, V. Kozlovsky, A. Loboda, V. Raznikov, I. Sulimenkov, A. Tolmachev, A. Kraft, H. Wollnik (1997): A new technique for decomposition of selected ions in molecule ion reactor coupled with ortho-time-of-flight mass spectrometry, Rapid Commun. Mass Spectrom. 11, 1649-1656; doi: 10.1002/(SICI)1097-0231(19971015)11:15<1649:AID-RCM67>3.0.CO;2-T.

[11] B. A. Mansoori, E. W. Dyer, C. M. Lock, K. Bateman, R. K. Boyd, B. A. Thomson (1998): Analytical performance of a high-pressure radio frequency-only quadrupole collision cell with an axial field applied by using conical rods, J Am Soc Mass Spectrom 9, 775-788; doi: 10.1016/S10440305(98)00042-7.

[12] A. Loboda, A. Krutchinsky, O. Loboda, J. McNabb, V. Spicer, W. Ens, K. Standing (2000): Novel Linac II Electrode Geometry for Creating An Axial Field in a Multipole Ion Guide, Eur J Mass Spectrom (Chichester, Eng) 6, 531-536; doi: 10.1255/ejms.383.

[13] B. A. Thomson (1998): 1997 McBryde Medal Award Lecture Radio frequency quadrupole ion guides in modern mass spectrometry, Can. J. Chem. 76, 499-505; doi: 10.1139/v98-073. 
[14] B. Brkić, N. France, A. T. Clare, C. J. Sutcliffe, P. R. Chalker, S. Taylor (2009): Development of quadrupole mass spectrometers using rapid prototyping technology, J Am Soc Mass Spectrom 20, 1359-1365; doi: 10.1016/j.jasms.2009.03.025.

[15] I. E. Dayton, F. C. Shoemaker, R. F. Mozley (1954): The Measurement of Two-Dimensional Fields. Part II: Study of a Quadrupole Magnet, Phys. Rev. 25, 485-489; doi: 10.1063/1.1771107.

[16] D. R. Denison (1971): Operating Parameters of a Quadrupole in a Grounded Cylindrical Housing, Journal of Vacuum Science and Technology 8, 266-269; doi: 10.1116/1.1316304.

[17] Gibson, Taylor (2000): Prediction of quadrupole mass filter performance for hyperbolic and circular cross section electrodes, Rapid communications in mass spectrometry RCM 14, 16691673; doi: 10.1002/1097-0231(20000930)14:18<1669:AID-RCM80>3.0.CO;2-\#.

[18] J. R. Gibson, S. Taylor (2001): Numerical investigation of the effect of electrode size on the behaviour of quadrupole mass filters, Rapid Commun. Mass Spectrom. 15, 1960-1964; doi: $10.1002 / \mathrm{rcm} .469$.

[19] Johannes Philipp Hoffrogge: A surface-electrode quadrupole guide for electrons. Dissertation, München, 2012.

[20] N. Sakudo, T. Hayashi (1975): Quadrupole electrodes with flat faces, Rev. Sci. Instrum. 46, 10601062; doi: 10.1063/1.1134394.

[21] C. G. Pearce, D. Halsall (1978): A quadrupole mass filter with flat electrodes, Int. J. Mass Spectrom. Ion Phys. 27, 31-41; doi: 10.1016/0020-7381(78)80099-0.

[22] T.-C. Chen, I. K. Webb, S. A. Prost, M. B. Harrer, R. V. Norheim, K. Tang, Y. M. Ibrahim, R. D. Smith (2015): Rectangular ion funnel: a new ion funnel interface for structures for lossless ion manipulations 87, 716-722; doi: 10.1021/ac503564c.

[23] K. Cheung, L. F. Velasquez-Garcia, A. I. Akinwande (2010): Chip-Scale Quadrupole Mass Filters for Portable Mass Spectrometry, J. Microelectromech. Syst. 19, 469-483; doi: 10.1109/JMEMS.2010.2046396.

[24] M. Geear, R.R.A. Syms, S. Wright, A. S. Holmes (2005): Monolithic MEMS quadrupole mass spectrometers by deep silicon etching, J. Microelectromech. Syst. 14, 1156-1166; doi: 10.1109/JMEMS.2005.851799.

[25] S. Boumsellek, R. J. Ferran (2001): Trade-offs in miniature quadrupole designs, J Am Soc Mass Spectrom 12, 633-640; doi: 10.1016/S1044-0305(01)00248-3.

[26] I. K. Webb, Garimella, Sandilya V B, A. V. Tolmachev, T.-C. Chen, X. Zhang, R. V. Norheim, S. A. Prost, B. LaMarche, G. A. Anderson, Y. M. Ibrahim, R. D. Smith (2014): Experimental evaluation and optimization of structures for lossless ion manipulations for ion mobility spectrometry with time-of-flight mass spectrometry 86, 9169-9176; doi: 10.1021/ac502055e.

[27] I. K. Webb, Garimella, Sandilya V B, A. V. Tolmachev, T.-C. Chen, X. Zhang, J. T. Cox, R. V. Norheim, S. A. Prost, B. LaMarche, G. A. Anderson, Y. M. Ibrahim, R. D. Smith (2014): Mobilityresolved ion selection in uniform drift field ion mobility spectrometry/mass spectrometry: dynamic switching in structures for lossless ion manipulations 86, 9632-9637; doi: 10.1021/ac502139e. 
[28] A. V. Tolmachev, I. K. Webb, Y. M. Ibrahim, Garimella, Sandilya V B, X. Zhang, G. A. Anderson, R. D. Smith (2014): Characterization of ion dynamics in structures for lossless ion manipulations 86, 9162-9168; doi: 10.1021/ac502054p.

[29] D. E. Austin, M. Wang, S. E. Tolley, J. D. Maas, A. R. Hawkins, A. L. Rockwood, H. D. Tolley, E. D. Lee, M. L. Lee (2007): Halo ion trap mass spectrometer, Anal. Chem. 79, 2927-2932; doi: 10.1021/ac062155g.

[30] G. Huber, T. Deuschle, W. Schnitzler, R. Reichle, K. Singer, F. Schmidt-Kaler (2008): Transport of ions in a segmented linear Paul trap in printed-circuit-board technology, New J. Phys. 10, 13004; doi: 10.1088/1367-2630/10/1/013004.

[31] D. Jiang, G.-Y. Jiang, X.-X. Li, F.-X. Xu, L. Wang, L. Ding, C.-F. Ding (2013): Printed circuit board ion trap mass analyzer: its structure and performance, Analytical chemistry 85, 6041-6046; doi: 10.1021/ac400864k.

[32] D. J. Douglas (2009): Linear quadrupoles in mass spectrometry, Mass Spectrom Rev 28, 937960; doi: 10.1002/mas.20249.

[33] A. Bekstein, M. Benhenni, M. Yousfi (2011): Collision cross sections and swarm coefficients of water vapour ion clusters ( $\mathrm{H} 2 \mathrm{O}) \mathrm{n} \mathrm{H}+$ with $\mathrm{n}=1,2$ and 3 in N2, $\mathrm{O} 2$ and air, Eur. Phys. J. D 61, 153-159; doi: 10.1140/epjd/e2010-10376-7.

[34] A. Bohnhorst, A. T. Kirk, S. Zimmermann (2016): Simulation aided design of a low cost ion mobility spectrometer based on printed circuit boards, Int. J. Ion Mobil. Spec. 19, 167-174; doi: 10.1007/s12127-016-0202-7.

[35] Florian Schlottmann, Maria Allers, Ansgar T. Kirk, Alexander Bohnhorst, Stefan Zimmermann (2019): A simple printed circuit board based ion funnel for focussing low $\mathrm{m} / \mathrm{z}$ ratio ions with high energies at elevated pressure, Under review.

[36] J. Langejuergen, M. Allers, J. Oermann, A. T. Kirk, S. Zimmermann (2014): High kinetic energy ion mobility spectrometer: quantitative analysis of gas mixtures with ion mobility spectrometry, Anal. Chem. 86, 7023-7032; doi: 10.1021/ac5011662.

[37] J. Langejuergen, M. Allers, J. Oermann, A. T. Kirk, S. Zimmermann (2014): Quantitative detection of benzene in toluene- and xylene-rich atmospheres using high-kinetic-energy ion mobility spectrometry (IMS), Anal. Chem. 86, 11841-11846; doi: 10.1021/ac5034243.

[38] A. T. Kirk, T. Kobelt, H. Spehlbrink, S. Zimmermann (2018): A Simple Analytical Model for Predicting the Detectable Ion Current in Ion Mobility Spectrometry Using Corona Discharge Ionization Sources, J Am Soc Mass Spectrom; doi: 10.1007/s13361-018-1970-6.

[39] A. T. Kirk, D. Grube, T. Kobelt, C. Wendt, S. Zimmermann (2018): A High Resolution High Kinetic Energy Ion Mobility Spectrometer Based on a Low-Discrimination Tri-State Ion Shutter, Anal. Chem. 90, 5603-5611; doi: 10.1021/acs.analchem.7b04586.

[40] J. de Gouw, C. Warneke, T. Karl, G. Eerdekens, C. Van Der Veen, and R. Fall, J. de Gouw, C. Warneke, T. Karl, G. Eerdekens, van der Veen, Carina, R. Fall (2003): Sensitivity and specificity of atmospheric trace gas detection by proton-transfer-reaction mass spectrometry, Int. J. Mass Spectrom. 223-224, 365-382; doi: 10.1016/S1387-3806(02)00926-0.

[41] M. M. Shahin (1966): Mass-Spectrometric Studies of Corona Discharges in Air at Atmospheric Pressures, J. Chem. Phys. 45, 2600; doi: 10.1063/1.1727980. 
[42] M. Pavlik, J. D. Skalny (1997): Generation of [H3O]+.(H2O)n clusters by positive corona discharge in air, Rapid Commun. Mass Spectrom. 11, 1757-1766; doi: 10.1002/(SICI)10970231(19971030)11:16<1757:AID-RCM16>3.0.CO;2-8.

[43] B. Cha, M. Blades, D. J. Douglas (2000): An Interface with a Linear Quadrupole Ion Guide for an Electrospray-Ion Trap Mass Spectrometer System, Anal. Chem. 72, 5647-5654; doi: 10.1021/ac0004862. 